\title{
I-DIGEST framework: towards authentic learning for indigenous learners
}

\author{
John Loewen ${ }^{1 *}$, Kinshuk ${ }^{2}$ and Jarkko Suhonen ${ }^{3}$
}

\author{
* Correspondence: \\ loewenj700@gmail.com \\ ${ }^{1}$ University of Eastern Finland, \\ 304-3800 Quadra Street, Victoria, BC, \\ Canada \\ Full list of author information is \\ available at the end of the article
}

\begin{abstract}
There is a distinct lack of authentic and relevant learning opportunities for Indigenous learners in formalized educational settings. This is due to multiple factors including the lack of incorporation of Indigenous knowledge into educational curriculum. A significant portion of Indigenous knowledge is in relation to the land and is experientially grounded. Therefore, any knowledge system used by Indigenous learners must take into account that learning is contextual to a place and should include a significant experiential learning component within the curriculum. Digital storytelling has emerged as a methodology for incorporating Indigenous knowledge into learning and with the literature identifying the lack of a formal curriculum design process, a framework is presented for incorporating digital storytelling into learning curriculum, allowing for authentic and relevant learning opportunities for Indigenous learners. To test the efficacy of our framework approach, prototype tools have been created to generate learning objects to be used in a case study course. Experts in the field of education and Indigenous knowledge were then asked to provide feedback on the efficacy of the framework. Strengths identified include the inclusion of knowledge holders and community members in the design process as well as providing a visual and oral alternative to the standard written method of expression. Weaknesses identified include possible lack of knowledge and support from educators as well as a lack of protocol to determining valid, relevant knowledge. Additional benefits identified include using this approach beyond the physical boundaries of a community, to allow, for remote community members to contribute to the knowledgebase.
\end{abstract}

Keywords: Indigenous knowledge, Educational technology, Framework, Learning objects, Digital storytelling, Mobile devices

\section{Introduction}

Indigenous learners worldwide have long-standing relationships with their environment. This knowledge of the environment and all of the relationships associated with it are often referred to as Indigenous knowledge. A significant proportion of Indigenous knowledge is directly related to the land (Hunter, 2005). For many Indigenous cultures, storytelling to preserve knowledge is an embedded way of life and has been around since time immemorial. Unfortunately, the educational systems currently encountered by many Indigenous learners are culturally irrelevant, leading to a distinct lack of enthusiasm for many of these learners worldwide (Battiste, 2002). Additionally, there is very little research in the domains of combining Indigenous knowledge and Western knowledge in a formalized way (Hartnell-Young \& Vetere, 2008). With the

(c) The Author(s). 2018 Open Access This article is distributed under the terms of the Creative Commons Attribution 4.0 International License (http://creativecommons.org/licenses/by/4.0/), which permits unrestricted use, distribution, and reproduction in any medium, provided you give appropriate credit to the original author(s) and the source, provide a link to the Creative Commons license, and indicate if changes were made. 
birth and spread of the Internet, Indigenous communities are beginning to realize how digital technologies can be used as a means documenting and preserving this knowledge (Srinivisan \& Huang, 2005). Cultural mapping (community-based mapping, participatory mapping) has emerged to bring alternative Indigenous and local perspectives to the attention of those who hold power and who control what usually appears on official maps (Crawhall, 2007). Storytelling is a powerful way in which to communicate experiences and to explore ideas (Skouge \& Rao, 2009). Srinvisan (2007) discusses the potential of participatory design to create ethnomethodological information architectures. In this context, ethnomethodology is the process of engaging a community to reflect on its practices. The shaping of these architectures allows for communities to serve as content creators, information architects, and ontology creators of their own systems. Following this line of thinking, Srinivasan (2007) believes that.

"a powerful direction for future information systems would follow a hybrid model wherein communities articulate their own information systems, yet on a meta-level multiple systems are integrated through global standards".

Without this approach, systems lack an organizational scheme that is reflective of the specific community.

With this in mind, the purpose of this study is to provide a framework that assists in allowing the incorporation of digital storytelling into learning curriculum with the goal to provide authentic and relevant learning opportunities to Indigenous learners. To initiate this process, a review of the literature is first provided and research gaps identified. The three main research questions evolving from the literature review and gaps analysis are presented and from these questions, we propose the I-DIGEST (InDIGEnous digital STorytelling) framework. The framework was instantiated by designing place-based tools for web-based and mobile devices to allow for the incorporation of digital storytelling into western Euro-centric curriculum by allowing the learners to create and share their own experiences and their relation to the curriculum. Mobile devices are included as part of the ICT approach as they allow learning to occur in the environment that the learner is most comfortable with, for example, on traditional territory. The framework and prototype tools were presented to Indigenous knowledge and curriculum experts who were then interviewed to provide feedback on the approach, and to identify whether they aided in solving the research problem. As the philosophical foundation of this research stems from a transformative worldview, a qualitative research approach was used (Creswell, 2013; Mertens, 2012) in the form of one-on-one interviews with participants to provide feedback on the efficacy of the approach in regards to solving the research problem. An analysis and discussion of the results is then provided and lastly, a lens on possible future directions of research is proposed.

\section{Review of the literature}

To lay a foundation for this research, the concept of experiential learning as it relates to Indigenous learners is reviewed, to highlight effective learning techniques that have been shown to be successful with Indigenous learners. From this position of knowledge, a particular method of learning, digital storytelling is positioned as an effective learning 
approach for Indigenous learners. Lastly, mobile technologies are introduced as they show promise as an effective tool for providing authentic learning opportunities for Indigenous learners.

\section{Experiential and collaborative learning in indigenous education}

Indigenous knowledge and thought is experientially grounded (Kawagley \& Barnhardt, 1998; Battiste, 2002). Often oral and symbolic, it is transmitted through the structure of Indigenous languages and passed on to the next generation through modeling, practice, and animation, rather than through the written word (Battiste, 2002). For Indigenous learners, McKnight et al. (2011) identify that an experiential approach to learning is an effective method for Indigenous learners. A deep awareness and understanding of the students' cultural backgrounds, life experiences and connections to the community are prerequisite for the development of appropriate pedagogical approaches that have the potential to connect learners to each other, their communities and the educative process (Nielsen et al., 2008). Digital storytelling has been identified as a methodology that can support this kind of approach (Skouge \& Rao, 2009). Digital storytelling makes use of a wide variety of techniques, ranging from standard storytelling in audio and video format as well as multimedia publications and shared events, for example, using collaborative software as a venue to interact (Skouge \& Rao, 2009). Digital storytelling is a way to celebrate the individual and the collective, and to lend respect and credence to the lived experiences of individuals through the collective co-creation of individual narratives, and provides participants with the opportunity to work together, tell and share stories, listen to others, and learn. For educators, digital storytelling provides an engaging way to bring lessons about community, culture, local values, and traditions into the classroom (Skouge \& Rao, 2009). An example of a useful implementation of collaborative learning implementing digital storytelling is provided by Duveskog \& Sutinen (2013) who utilized digital storytelling platform to create localized content to assist in AIDS/HIV education and counselling for Tanzanian students. Their research showed that by incorporating real-life stories of the students that incorporate cultural relevance, ownership and a story-based approach, barriers to learning (for example, theoretical, cultural, pedagogical, and technical) are reduced, thus enhancing the learning process.

There are some issues regarding experiential learning approaches such as digital storytelling. For example, many teachers who teach within Indigenous communities are non-Indigenous. According to McKnight et al. (2011), it is known that non-Indigenous teachers should develop an understanding of Indigenous ways, but the knowledge is currently presented didactically through lectures and tutorials in pre-service education subjects, not in an experiential form. Additionally, such approaches put additional burden on teachers and course designers who have to create additional learning materials to support the learning process. To this end, ICT tools may provide ways in which to ease the burden, for example, to provide a way to store, index, and re-use experiential learning objects, such as digital stories. Raseroka (2008) identifies that the development of ICT is a significant tool for the capture of predominantly oral-based Indigenous knowledge to facilitate both its preservation and access beyond person-to-person communication. 


\section{Learning on the land - mobility for knowledge acquisition}

Given that most underserved Indigenous people (for example, those in remote, rural communities) lack adequate internet access, the delivery of information using an inexpensive mobile device is a good option (Kim, 2009). Naismith et al. (2004) state that we need $\mathrm{m}$-learning that places the student at the center of the learning process, promotes collaboration, provides an understanding of the authentic context of knowledge, and encourages independent learning behavior, so that students become lifelong learners. Dyson et al. (2009) discuss how mobile devices may enhance active, experiential learning, specifically mobile supported fieldwork. As identified previously, this methodology works well with Indigenous learners as it can allow for learning to take place in the environment that the learner is comfortable in, for example, out on traditional harvesting and hunting grounds using devices that support experiential learning (for example video, audio, and camera) in this kind of informal environment. Additionally, mobile devices may provide community members with access to these materials while remaining in their own community.

\section{Research questions}

With the growth of digital storytelling as a methodology of experiential learning arises the critique of how these knowledge artifacts are categorized and stored. Whose categorization is being used? For example, is this built around a system of logic that follows traditional Eurocentric notions of rationality? These are systems that do not seem to primarily be concerned with the community or specific cultural group's authorship, epistemology, or ontology (Srinivasan, 2007). Although educational uses of digital storytelling have been conducted in specific countries, there is limited information about the current situation of using digital storytelling in education throughout the world (Yuksel et al., 2011). As recently as 2012, critical studies of digital technology use among Indigenous communities remained largely undeveloped (Srinivasan, 2012). If we perform a search into the research of Indigenous learning and digital storytelling since 2012 (in Google Scholar) we will find more than 3000 articles. However, if we narrow the focus to incorporate the concepts of decolonization, reconciliation, and transformative learning, gaps begin to appear. An example of research that fits this narrower focus is that of Castleden et al. (2013) who research the effectiveness of digital storytelling as a component of a transformative learning approach, both for non-Indigenous and Indigenous learners. Digital storytelling may be used as a method to incorporate components of transformative learning, namely experiential learning and journal writing, into the learning process. The authors found digital storytelling in this context as a useful tool for evaluating students' progress in transforming their worldviews. What is still missing is identified by Poitras Pratt and Lalonde (2016) who identify that for technology to further the work of decolonization and reconciliation, we must envision new landscapes of learning, identifying tool creation within a learning management system that "enables educators to create an educational environment where traditional ways of knowing, being, and doing are foundational aspects in educational programming".

Using these gaps as a starting point and experiential learning at the forefront, the following research questions emerged:

- To what extent are Indigenous educators able to integrate digital storytelling into learning using ICT tools such as a learning management system (LMS)? 
- As mobile devices allow the learner to be immersed within the environment, how may they be used to incorporate digital storytelling into education to provide relevant and authentic learning opportunities for Indigenous learners?

- What are educators' and Indigenous knowledge experts' views and concerns on the I-DIGEST framework as a methodology for integrating digital storytelling into learning curriculum for Indigenous learners?

The significance of the study is that it provides insight into ways in which Indigenous learners may combine digital storytelling with place-based thinking by connecting stories to curriculum as well as to a place on a graphical, interactive map within a formalized learning environment. For example, once all learners in a cohort have created and shared their stories on the map, other learners may "walk" around the landscape to view, comment and to learn about the stories created by others, thus creating a standardized collaborative and authentic learning environment.

\section{I-DIGEST framework}

Towards the goal of providing authentic relevant learning opportunities for Indigenous learners, we propose the I-DIGEST framework. The I-DIGEST framework is shown in Fig. 1.

The 5 main phases of the I-DIGEST framework are as follows:

1. Knowledge gathering - With digital storytelling, knowledge gathering consists of creating multi-media content from various sources such as audio, video and image files. Digital storytelling is the process of illustrating personal narratives and stories with artwork, music, voice-overlay, video-clips and text (like a first-person mini-movie). Knowledge holders, knowledge sharers and community leaders play an integral role in the knowledge generation process, which in order to acquire a depth of knowledge, is time-consuming and intensive (Srinivasan, 2007).

2. Knowledge building - Once the digital stories have been created and entered into the system (referred to as the Digital Story Repository in Fig. 1), they may be used within relevant curriculum by associating digital stories with curriculum thereby providing authenticity and relevancy to learning. This knowledge may be managed within the course and/or managed locally within each community, ensuring that the stories are shared appropriately.

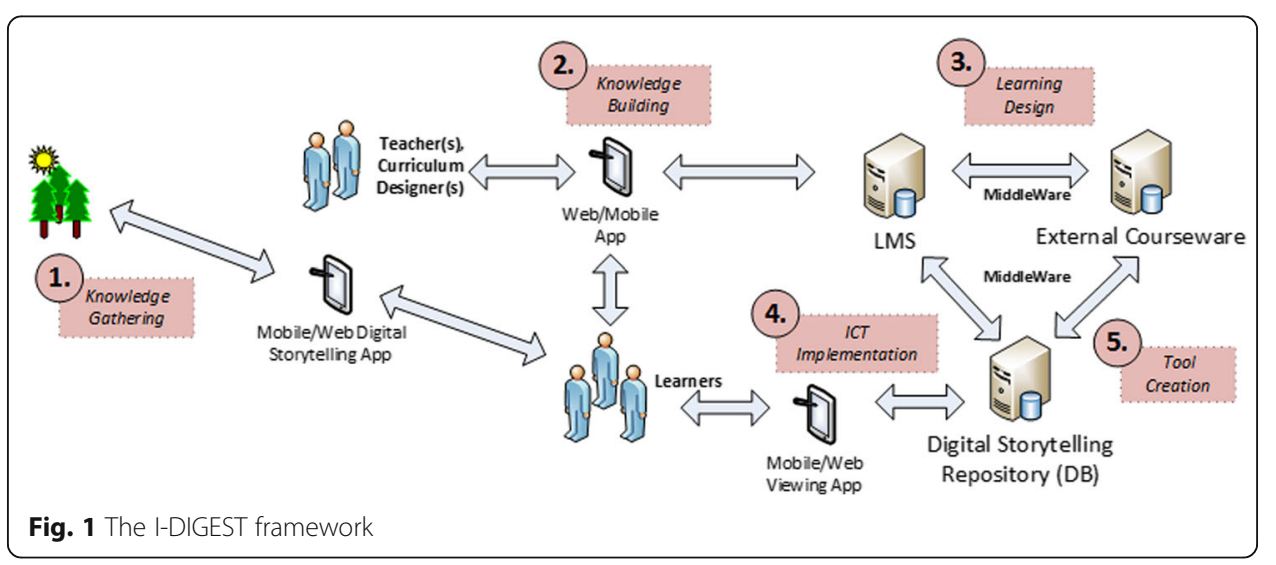


3. Learning Design - the digital stories may then be converted to learning objects conforming to a standard format, such as IMS-LD or SCORM, allowing for reusability. This phase runs parallel with the ICT Implementation phase as tools may be created to simplify the input process for learning objects, allowing for their storage, association, and retrieval within the learning environment.

4. ICT Implementation - ICT tools may be designed in order to simplify the creation and addition of digital stories and to encourage place-based learning to take place within informal and formalized curriculum. For example, a fundamental issue identified in previous research was that any tool used in the gathering of knowledge must not take away from opportunities for actual place-based learning (Loewen et al. 2017). Mobile devices have been identified as a useful tool to allow for knowledge gathering and input into a learning system (Dyson et al., 2009), allowing for learning to take place in the environment that the learner is most comfortable in. In Fig. 1, mobile devices may be leveraged to create digital stories (i.e. mobile/web digital storytelling app) and also to view them (i.e. mobile/web viewing app). Figure 2 provides a more in-depth visual representation of this process. The learner may be on the land, at a point of interest, for example, a monumental cedar tree and may find more information about this tree through the app, or may provide additional information,for example personal experience in the form of video, audio, text, or images.

5. Tool Creation - Tools must be created, in the form of web-based and mobile interfaces, to allow for the input of knowledge, as well as for the access of knowledge using a place-based approach, for example, by identifying digital stories and associated learning objects by location (i.e. using GPS co-ordinates).

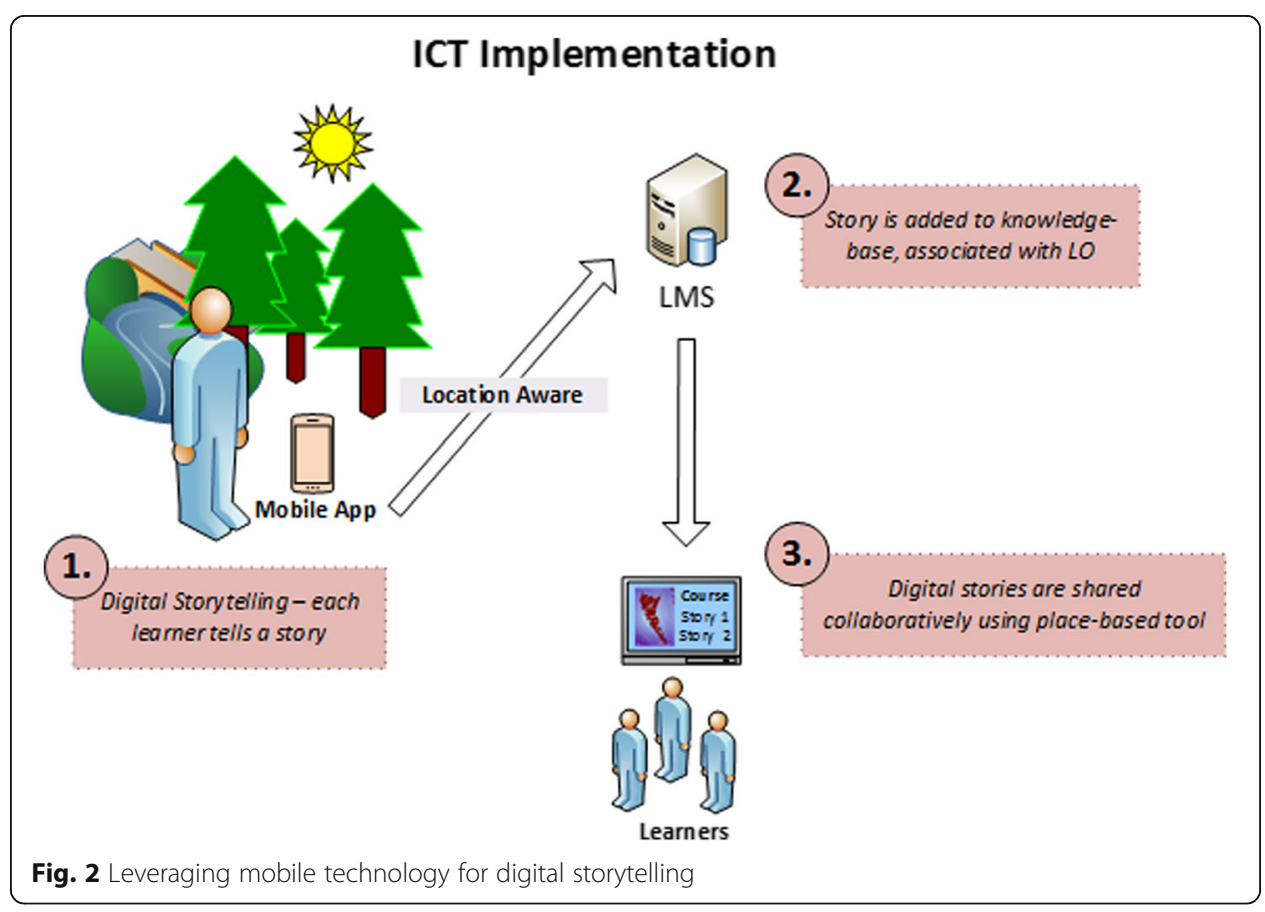


In order to prove the efficacy of the I-DIGEST framework, we created a prototype set of applications to be applied to a case study scenario. Feedback on this approach was provided via a video presentation and questionnaire to educational and Indigenous knowledge experts. For standardization and reusability in mind, all of the tools have been created using the IMS Learning Tool Interoperability (IMS-LTI) extensions to allow for centralized management of all of the knowledge and information within the LMS.

\section{Prototyping the framework}

To show proof of concept of the I-DIGEST framework, the following set of LMS tools were created:

- Learning Object Creation Tool - allows for the creation of a Learning Object (LO) or objects that are then added to the LMS database. The database gives each LO an ID, allowing for the association of digital stories (and possibly other types of LOs) at a later time. This tool has been created to conform to the IMS Learning Design (IMS-LD) learning object standard for the purpose of standardization and reusability.

- Digital Storytelling Interface - allows for a user of the system (learner, teacher, etc) to create a digital story and associate it with a place on a map, as well as with already created curriculum. This interface may be used in two different ways: firstly via a visual web-based interface (for example on a map), or secondly, via a mobile device, which provides the opportunity for the story teller to create stories from within the environment that they are most comfortable.

- Collaboration tool - once a user has created a story, the collaboration tool allows users to share it to individual users or to a group of users (for example, to other learners in the course, to a cohort, or to a community).

- Visual Place-based viewing interface -Allows teachers and other learners to navigate the map to view digital stories created by other users. This may be done through a visual map within the learning environment or via mobile devices, which provide additional functionality by allowing the learner and teacher to navigate to the actual physical location where the digital story was originally created, thus providing a more authentic, relevant, and potentially interactive environment in which to learn.

To further test the efficacy of the I-DIGEST framework, a case study implementing prototype tools was provided for expert analysis. A very simple example course was created and each of the prototype tools was used to demonstrate the functionality of the I-DIGEST framework within the example course. This was accomplished by firstly, creating a set of LOs; secondly, incorporating experiential learning by creating a digital story that was associated with a learning object; thirdly, sharing the story with other learners, and lastly, viewing a digital story created by another learner.

\section{Plants of Haida Gwaii - A case study}

The Haida people have resided on Haida Gwaii for millennia. Located at the edge of Western Canada, Haida Gwaii is home to some of the last remaining tracts of coastal temperate rainforest. Over the centuries, the Haida people have relied on the ocean and forests for their material, cultural, and spiritual well-being. Since the 1980s, Haida resistance to 
multi-national logging efforts has lead to the revitalization of culture and community and in western terms, greater local control of the land through community-based forest tenures and legal recognition of Haida title to the land (Takeda, 2014). Education that includes local knowledge, insight and perspectives within the curriculum may further assist in the revitalization process. With this in mind, the course created is called "Plants of Haida Gwaii", and the initial course structure is shown in Fig. 3.

A learning object was created for each type of plant and consisted of knowledge gleaned from Euro-centric resources. Indigenous learners have the opportunity to view this knowledge, to comment on it, and to discuss it. To allow for experiential and place-based learning opportunities, the learner may then use digital storytelling to augment learning objects by using the digital storytelling interface tools described previously. The basic interface for adding a digital story is shown in Fig. 4.

When creating a digital story, the learner has the choice of creating it from this web interface by navigating to a physical location using a mobile device that is GPS enabled. The two interfaces for creating a digital story are shown in Fig. 5.

The output of this process is a digital story that provides local knowledge, perspective and insight into the importance of each of these plants for the well-being of local people in a material, cultural and spiritual context. The digital story may then be associated with the learning object already created for that plant in the course curriculum. Once the learners have created their stories, they become part of the knowledge base for the course and may be shared for access by other learners through the same web-based interface and mobile device app used for the creation of the stories.

\section{Research design}

Stemming from the philosophical foundations of a transformative worldview, this research follows a qualitative design approach, and uses the one-on-one interview research methodology, identified by Creswell (2013) as an effective qualitative technique for this approach. One-on-one interviews were performed with education and Indigenous knowledge experts, either in person or by email. Participants were asked to view a 20 min explanation of the I-DIGEST framework and were given a demonstration of the

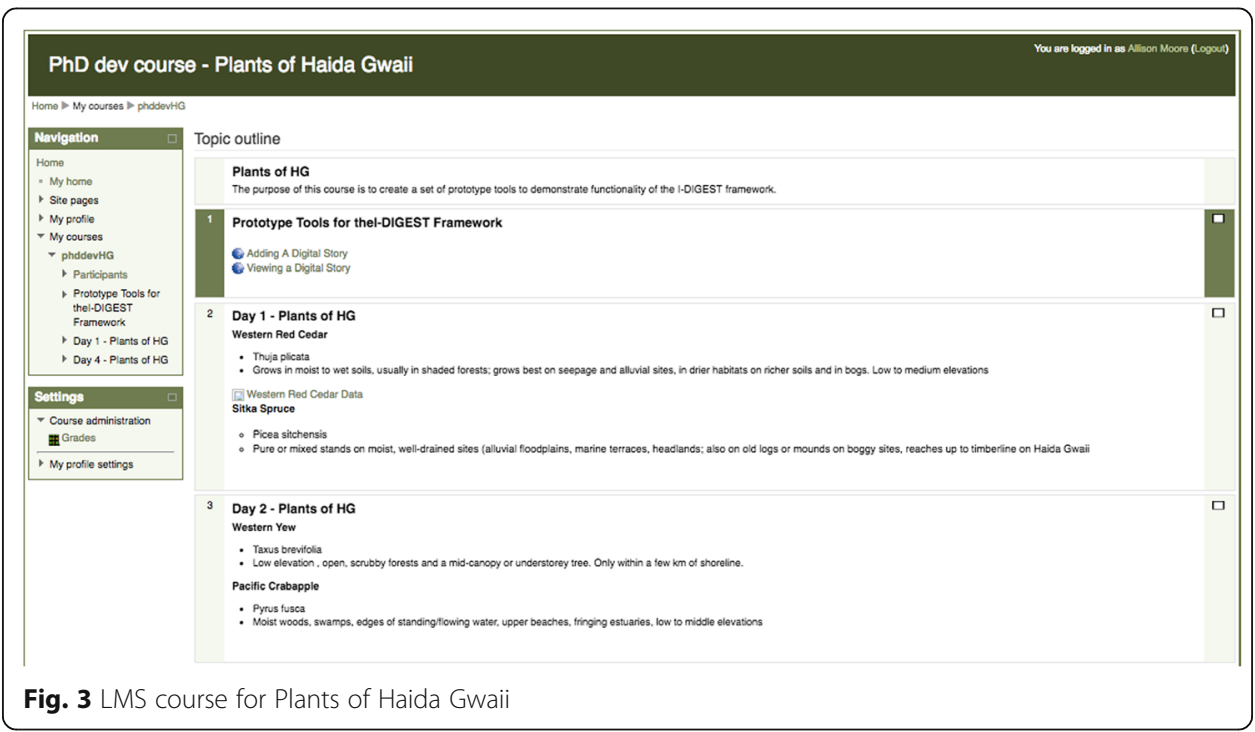




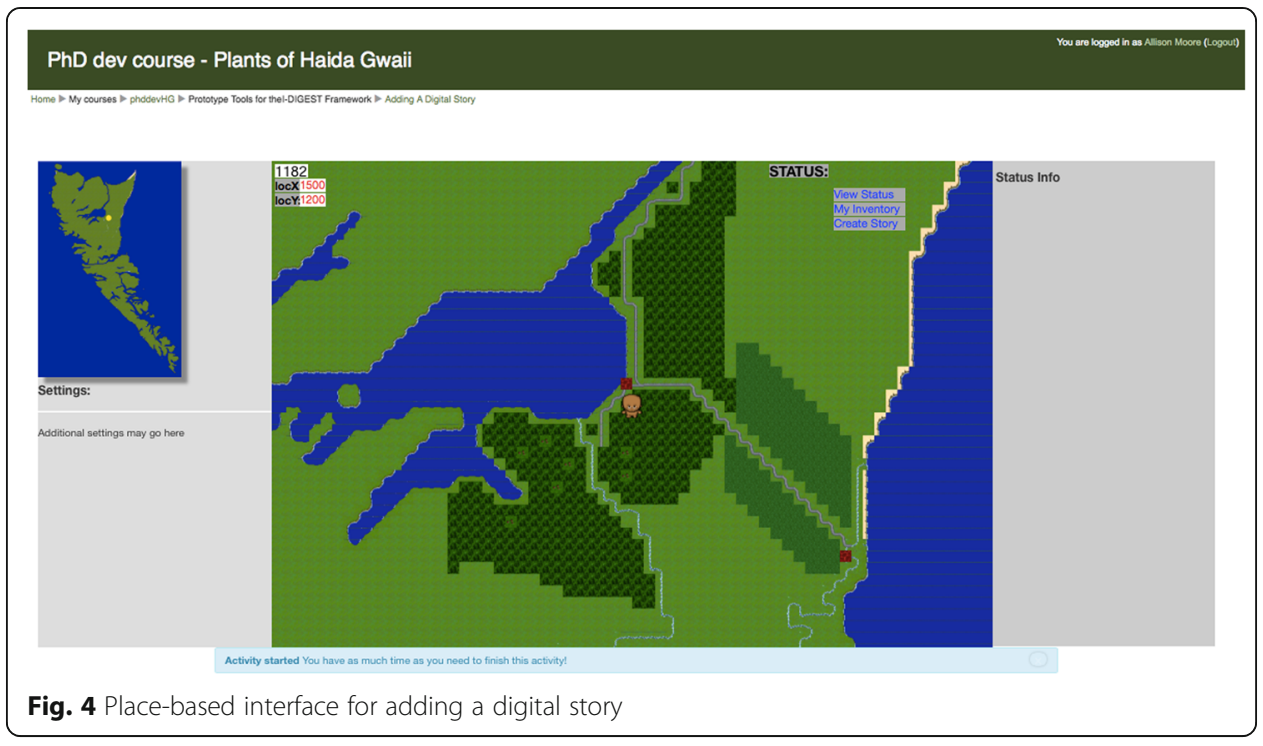

case study example that implemented the prototype tools. Participants were then asked the following questions:

1. Please identify your professional roles and interests as well as any other affiliations and roles you would like to mention.

2. In your position as an educator, teacher or in any supporting role, have you incorporated digital storytelling into learning? If yes, as best you can, please describe how. If not, please identify reasons why you have not.

3. Do you incorporate any mobile device technologies in your classroom? If yes, please describe how. If not, please identify why you have not

4. What do you see as the benefits/drawbacks of this system for the learner/teacher/ curriculum designer in regards to providing authentic learning opportunities for Indigenous learners?

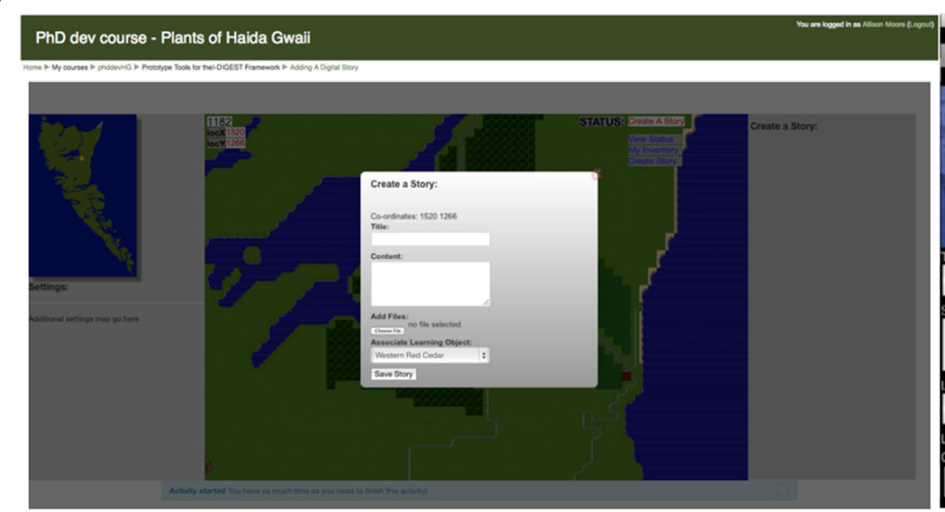

a

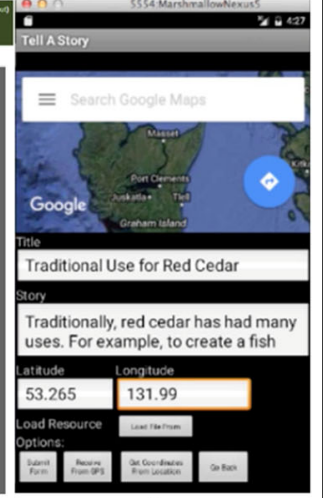

b

Fig. 5 Methods for adding a digital story to a course using; (a) web interface, (b) mobile app interface; ICT - Information and communications technology, IK - Indigenous knowledge, IMS-LD - IMS learning design, IMS-LTI - IMS Learning tool interoperability, LMS - Learning management system, LO - Learning object, SCORM - shareable content object reference model 
5. What do you see as the overall strengths/weaknesses of this approach to designing learning for Indigenous learners?

6. Are there other ways in which you could envision a system like this being used? Please elaborate if possible.

Each question was designed to best elicit responses that fit the research questions, specifically the extent in which participants integrate digital storytelling, whether participants use mobile device technologies and how, and lastly, what their views and concerns are regarding digital storytelling as a learning tool used in this approach.

\section{Participants}

From a pool of approximately 20 experts, 10 participants provided feedback for this study. To provide perspectives from both world views, both Indigenous (5) and non-Indigenous (5) experts were included in the study.. All participants chosen were highly qualified in the education and educational technology sectors including the following areas of expertise:

- Faculty Development, Center of Excellence for Teaching and Learning (tertiary institution)

- Director of Aboriginal Education and Community Connections (tertiary institution)

- Managing Editor, web and paper for an Indigenous nation

- PhD, Faculty of Business, Indigenous Leadership, Human Resource Management and Leadership, and Indigenous Governance (tertiary institution).

- Curriculum and Research Development, Indigenous Governance

- Indigenous Studies Faculty, Indigenous Elders Coordinator (tertiary institution)

- Indigenous Advisor (tertiary institution)

\section{Results}

Working from the questions posed to the participants, and on the richness of the data, particularly for questions 4 and 5, the results were coded into three themes (Creswell, 2013); strengths, weaknesses, and alternative uses.

\section{Strengths}

Strengths identified for the I-DIGEST framework and experiential learning approach include, firstly, that it builds an inclusive and respectful process for knowledge sharing. As one participant stated:

"Including learner voice in the design and then evaluation strengthens the modelfeedback loops throughout the process could be easily integrated".

This supports the design of phase 1 of the I-DIGEST framework as it may incorporate learners, knowledge holders and community members into the design process. As one participant stated, it is important that: 
"the local community and Indigenous knowledge keepers are included in the curriculum design to help create culturally safe boundaries for Indigenous learners."

Another expert identified the I-DIGEST framework as.

"allowing us (Indigenous peoples) the opportunity to share our stories as appropriate vs. stories being told 'about' us."

Secondly, as noted by Srinivasan \& Huang (2005), there is promise in approaches that engage communities to create and document information about their lives and collectively reflect on this. To further support this, another participant stated that the system:

"allows the students to tap into what engages them, and to bring it back to the theory, because every learner comes in with their unique perspective".

Being able to provide a unique perspective and integrate it into the learning process by associating it with curriculum is the foundation of phase 2 of the I-DIGEST framework (shown in Fig. 1). Thirdly, from an Indigenous perspective, particularly one that historically uses oral traditions to pass on knowledge from generation to generation, this kind of tool allows an alternative path of expression in learning:

"From an Indigenous perspective, you have these ideas and thoughts, you're not that skilled as a writer to get it across; the visual medium allows you to".

The experiential learning methodology of digital storytelling has been identified as being a method allowing Indigenous learners to expand the reach of traditional stories, using a visual platform to maintain oral tradition (Duveskog, Tedre, Sedano \& Sutinen, 2013). Lastly, a standardized approach, identified in previous research as warranting further research (Sen, 2005; Hartnell-Young \& Vetere, 2008), is also seen as valuable by participants as it allows "access for a wider audience, and therefore more accessibility", lending credence to phase 3,4 and 5 of the I-DIGEST framework, where ICT approaches have been designed and tools created with standardization in mind, allowing for learning object accessibility and reusability.

Lastly, multiple participants identified the mobile device integration of the I-DIGEST framework as very important and novel as many rural Indigenous communities lack traditional ICT infrastructure and have moved straight to mobile devices. Therefore any solution that incorporates mobility will reach a much larger portion of the rural Indigenous audience.

\section{Weaknesses}

Ensuring the right knowledge experts and teachers are involved in the design and delivery process is seen as a very difficult task and hence should be considered a weakness. As one participant states:

"Traditional protocols and teachings will need to guide this process of knowledge sharing and curriculum development". 
This is further supported by Bang et al. (2013) who observe that the design of learning environments and the development of technologies must centrally involve Indigenous communities as decision makers. Referring back to the literature, the lack of training in cultural diversity has been identified as an issue (Philpott et al., 2009), and warrants further research into ways in which the problem may best be approached and alternative solutions found. This was supported by multiple participants, with one stating that some educators are not willing to change:

"There's this old, traditional 'this is the way you were taught, this is the way I was taught and this is the way it will be taught', still that really traditional 'wordy' model".

Lastly, privacy, ethics, and security issues have also been identified as an area of concern by participants:

"I worry that Indigenous sacred places could be identified and then visited by people who shouldn't be there ".

Again, this is supported by the literature (Raseroka, 2008; Sen, 2005) due to the possibility of misappropriation and misuse of this material. Any implementation that follows the I-DIGEST framework must carefully consider the audience and the usage of the content created.

\section{Alternative uses}

Some alternative uses were identified by participants. Firstly, it was mentioned by one participant that this sort of system would be useful for international students, who in the participant's experience, enter the learning environment feeling excluded from the start. Any tool that can allow them to incorporate their own perspective, their own story into the curriculum, "can assist in this process". Multiple participants identified that this form of learning is also vital to the language revitalization process. Secondly, one participant identified a recent exhibit at an art gallery, where a First Nations community in Canada explored how place is re-assigned importance, as being a useful medium for incorporating the I-DIGEST framework.

"I envision this platform providing an authentic learning experience for Indigenous students of all ages."

This participant is referring to the ability of the I-DIGEST tools to allow for the creation of individual digital stories that may compliment this exhibit by providing place-based associations for each narrative, thereby providing a richness of data that was not previously shared. Another participant observed that.

"Indigenous Nations wanting to rebuild their knowledge systems and connection to the land could benefit from a system like this."

It was identified that there should be a way in which to create digital stories from urban environments, to be able to tell a story from afar, not just from within the 
traditional territory of an Indigenous community, thereby allowing relocated community members to re-connect with and to contribute to their community and their culture in an authentic and relevant way.

\section{Discussion}

The purpose of this research was to find ways in which ICT may be leveraged to create authentic and relevant learning opportunities for Indigenous learners. The literature identified experiential learning as an effective methodology for Indigenous learners, for example, digital storytelling. It is worth noting that digital storytelling is not the only methodology that may be implemented using the I-DIGEST framework. Author et al. (2016) used a fuzzy logic approach within a similar framework, the I-SLATE framework. The first research question asked Indigenous educators to what extent they were able to integrate digital storytelling into learning using ICT tools such as an LMS. Participants identified that digital storytelling builds an inclusive and respectful process for sharing knowledge in a way that for example, fits within a culture that historically uses oral traditions, quite often in the form of stories, to pass on knowledge from generation to generation.

The second research question asked participants about their views and concerns regarding integrating digital storytelling into learning curriculum for Indigenous learners. Overall, participants identified that the approach was novel and interesting but voiced concerns that it would require further iterations to overcome some of the trepidation that inherently comes with new approaches to learning, particularly those that involve ICT approaches. For example, a participant requested more detail on how the ICT components of the approach would ensure that privacy and security of the knowledge was maintained.. Bang et al. (2013) observe that as revitalization and regeneration in Indigenous communities continue, it will become increasingly necessary to repatriate technologies that are for new knowledge building as well as for knowledge dissemination and sharing. This will require that the design of learning environments and the development of technologies must centrally involve Indigenous communities as decision makers. Feedback from participants indicates that this research provides solutions towards this goal, for example, by including local knowledge holders and community members in the design process, and by incorporating oral and visual methods of expression into the knowledge building process. From the transformative worldview employed in this research approach, these processes may be used to challenge and subvert the constraints imposed on Indigenous learners, for example, in the form of a colonialist oppressive educational system (Mertens, 2012). From this, it is clear that moving forward, any educational initiative that involves Indigenous learners in a community must fully involve the Indigenous knowledge keepers and the Elders within the community in the process of creating and vetting any knowledge, including digital stories about that community. Additionally, any external influences to this process, including educators and curriculum experts, must have the knowledge, the expertise, and most importantly, the trust of the community to support the digital story creation process.

Addressing the third research question of how may mobile devices allow the learner to be immersed within the environment in which they are comfortable, to allow for the incorporation of digital storytelling into the curriculum for Indigenous learners, it was identified that mobile devices allowed for place-based narrative, allowing the learner to 
be immersed within the environment they are most comfortable with. This methodology also allows the learner to share knowledge using the form that they are most familiar with for example, as an oral representation rather than a written representation. Additionally, due to the lack of ICT infrastructure in many rural Indigenous communities and the increasing prevalence of mobile technologies in these same communities, the mobile device integration of the I-DIGEST framework allows for learning opportunities to reach a much larger proportion of the rural Indigenous audience.

The novelty of this research is that it provides a standardized approach towards providing authentic and relevant learning for Indigenous learners. This standardized methodology is missing in previous research; the I-DIGEST framework moves towards standardization through the creation of ICT tools and approaches that allow for the incorporation of personal narratives, in the form of digital storytelling, into pre-created curriculum. The significance to these observations and findings is that if authentic learning is desired, it provides credence to the framework of designing learning with community involvement at the forefront. These steps cannot be skipped, and therefore any educational path taken by educators from outside to within an Indigenous community is a lengthy, consultative, careful, considerate, and respectful journey. It is worth mentioning that this system has been devised with Indigenous learners at the forefront, but there is no reason why this system may not be used in other environments where place-based learning environments provide an effective method of learning. Additions suggested by participants include providing an outline regarding how to tell a story and the key components of storytelling from an Indigenous perspective for learners before they embark on adding content. It cannot be assumed that all students are technically-savvy and innately know what creating a digital story entails, particularly some of the issues and concerns that are raised regarding privacy, security and ethics from within and beyond a community.

\section{Conclusions and future work}

As an experiential learning methodology, digital storytelling provides an engaging way to bring lessons about community, culture, local values, and traditions into the classroom (Skouge \& Rao, 2009). The main purpose of this study is to provide a framework that assists in allowing the incorporation of digital storytelling into learning curriculum with the goal to provide authentic and relevant learning opportunities to Indigenous learners. The I-DIGEST framework was designed and a relevant course example and prototype learning approach were presented to expert participants to illustrate how the I-DIGEST framework may be used in practice. Issues identified in previous literature include the initial setup of the framework and of the knowledge data, which are both labor and time intensive and to this end, additional tools have been created to streamline this process. Additionally, there may be resistance from communities with any ICT tools and initiatives that take away from opportunities for place-based learning to occur on traditional lands (Loewen et al. 2017). What has become clear in the validation of this approach, particularly from Indigenous expert feedback, is that any tool used to augment learning must build on the principles of supporting learners to engage directly with their environment and their community. The benefits to the I-DIGEST framework are, for example, that it allows Indigenous learners to stay connected to the land, to follow a place-based pedagogy, while providing an opportunity to incorporate digital 
storytelling into established curriculum, through a standardized methodology thereby providing authentic and relevant learning opportunities. Additionally, the I-DIGEST framework and tools allow for learners within the course to collaborate with each other by providing a common place-based interface in which to share their stories. Further to this, if a modular approach is taken and the proposed framework is followed, then once the knowledge building exercises are completed, libraries may be created, used and added to in future iterations. Lastly, place-based tools such as this may assist in showing land use patterns which may have the important benefit of showing how a community or communities access the land and resources within their traditional territories. For example, this type of knowledge may be useful to refer to when discussing land and title for Indigenous communities worldwide. One casual observer of the framework and tools commented that this sort of approach would have been very useful during the 2010 northern British Columbia Enbridge Northern Gateway Project Joint Panel proceedings as a tool to highlight how the land and resources were used and intertwined within and between the Indigenous (and non-Indigenous) communities affected by the proposed pipeline. This knowledge could potentially be used as a way in which to educate non-Indigenous peoples and other communities on Indigenous peoples' land usage needs and patterns.

There are a few recognized limitations to this study. Firstly, as a qualitative study, the sample size is small relative to quantitative research. A pool of 20 expert participants were considered and only 10 responded. Ideally the percentage of respondents would have been higher. Secondly, access to communities was an issue due to the length of time needed, location, and the manner in which it takes to establish relationships and trust within the community. Lastly, due to geographical, time, and cost restraints, the questions were elicited in a questionnaire format. A more interactive interview process may have provided a richer set of resulting data.

Future work of interest may include research on additional areas of application for this framework, for example, using the tool as a way in which to impart knowledge to the educators of the learners in the I-DIGEST framework, to help them better understand how Indigenous learners interact with their community and the environment. Additionally, taking the I-DIGEST framework to a community or communities and applying the tools and concepts towards the creation of digital stories to compliment learning would provide more empirical data to further validate the I-DIGEST framework.

\section{Acknowledgements}

Thanks to Dr. Kinshuk and Prof. Jarkko Suhonen for their guidance on writing this paper.

Authors' contributions

The main author $(\mathrm{J} L$ ) performed all research and writing of the paper. $\mathrm{K}$ and $\mathrm{S}$ provided insight and editing of the paper. All authors read and approved the final manuscript.

Competing interests

The authors declare that they have no competing interests.

\section{Publisher's Note}

Springer Nature remains neutral with regard to jurisdictional claims in published maps and institutional affiliations. 
Received: 25 February 2018 Accepted: 21 May 2018

\section{Published online: 01 June 2018}

\section{References}

J Loewen, Kinshuk, J Suhonen. I-SLATE: Designing a culturally relevant framework for authentic learning. Smart. Learn. Environ. 4(1), 9 (2017)

M Bang, A Marin, L Faber, ES Suzukovich, Repatriating indigenous technologies in an urban Indian community. Urban Educ. 48(5), 705-733 (2013)

M Battiste, Indigenous knowledge and pedagogy in First Nations education: A literature review with recommendations (Apamuwek Institute, Ottawa, 2002)

H Castleden, K Daley, V Sloan Morgan, P Sylvestre, Settlers unsettled: Using field schools and digital stories to transform geographies of ignorance about indigenous peoples in Canada. J. Geogr. High. Educ. 37(4), 487-499 (2013)

N Crawhall, in Reflection paper presented for the UNESCO Division of Cultural Policies and Intercultural Dialogue in 2007. The role of participatory cultural mapping in promoting intercultural dialogue We are not hyenas (2007)

J Creswell, Research Design: Qualitative, Quantitative, and Mixed Methods Approaches (Sage Publishing, London, 2013)

M Duveskog, E Sutinen, Enriching student HIV awareness by digital storytelling. J. Educ. Multimed. Hypermed 22(4), 383-406 (2013)

LE Dyson, A Litchfield, E Lawrence, R Raban, P Leijdekkers, Advancing the M-Learning Research Agenda for Active, Experiential Learning: Four Case Studies. Aust. J. Educ. Technol. 25(2), 250-267 (2009)

E Hartnell-Young, F Vetere, A means of personalising learning: Incorporating old and new literacies in the curriculum with mobile phones. Curric. J. 19(4), 283-292 (2008)

J Hunter, The role of information technologies in indigenous knowledge management. Aust. Acad. Res. Libr. 36(2), 109-124 (2005)

AO Kawagley, \& R Barnhardt (1998). Education indigenous to place: western science meets native reality. Retrieved from: https://eric.ed.gov/?id=ED426823

PH Kim, Action research approach on mobile learning design for the underserved. Educ. Technol. Res. Dev. 57(3), 415435 (2009)

AD McKnight, G Hoban, W Nielsen, Using Slowmation for animated storytelling to represent non-aboriginal preservice teachers' awareness of" relatedness to country". Australas. J. Educ. Technol. 27(1), 41-54 (2011)

DM Mertens, Transformative mixed methods: Addressing inequities. Am. Behav. Sci. 56(6), 802-813 (2012)

L Naismith, P Lonsdale, GN Vavoula, M Sharples, Mobile Technologies and Learning (Futurelab. University of Birmingham, Birmingham, England, 2004)

WS Nielsen, C Nicol, J Owuor, Culturally-responsive mathematics pedagogy through complexivist thinking. Complicity: An Int. J. Complexity Educ. 5(1), 33-47 (2008)

D Philpott, D Sharpe, R Neville, The effectiveness of web-delivered learning with aboriginal students: Findings from a study in coastal Labrador. Canadian Journal of Learning and Technology/La revue canadienne de l'apprentissage et de la technologie 35(3) (2009)

Y Poitras Pratt, S Lalonde, in Proceedings of the IDEAS: Designing for Innovation, pp. 111-122. Designing and Sharing Relational Space through Decolonizing Media (University of Calgary, Calgary, 2016)

K Raseroka, Information transformation Africa: Indigenous knowledge-securing space in the knowledge society. Int. Inf. Libr. Rev. 40(4), 243-250 (2008)

B Sen, Indigenous knowledge for development: Bringing research and practice together. Int Inf Libr Rev 37(4), 375-382 (2005)

JR Skouge, K Rao, Digital storytelling in teacher education: Creating transformations through narrative. Educ. Perspect. 42, 54-60 (2009)

R Srinivasan, Ethnomethodological architectures: Information systems driven by cultural and community visions. J. Am. Soc. Inf. Sci. Technol. 58(5), 723-733 (2007)

R Srinivasan, Rethinking digital cultures and divides: The case for reflective media. Inf. Soc. 28(1), 24-36 (2012)

R Srinivasan, J Huang, Fluid ontologies for digital museums. Int. J. Digit. Libr. 5(3), 193-205 (2005)

L Takeda, Islands' Spirit Rising: Reclaiming the Forests of Haida Gwaii (UBC Press, Vancouver, 2014)

P Yuksel, BR Robin, \& S McNeil (2011). Educational uses of digital storytelling around the world. In Proceedings of Society for Information Technology \& Teacher Education International Conference (pp. 1264-1271). 